\title{
ALTERnative Familie - Gastfamilien für Seniorinnen und Senioren
}

\author{
Astrid Henriksen \\ Mitglied der Kommission Recht der sozialen Sicherung, Famili- \\ enlastenausgleich des djb; Sozialamtsleiterin der Stadt Bremer- \\ haven
}

\section{Gastfamilien}

Die durch den demografischen Wandel zunehmende Anzahl von überwiegend weiblichen älteren, an Demenz leidenden und pflegebedürftigen Menschen in Verbindung mit dem Wunsch der Pflegebedürftigen, möglichst lange in der eigenen Wohnung zu bleiben, stellen die Gesellschaft, die Betroffenen selbst, deren überwiegend weibliche pflegende Angehörige und die Sozialleistungsträger zukünftig vor eine erhebliche Herausforderung. Hier sind innovative Alternativen zu den bisherigen Versorgungsstrukturen gefragt.

Das Modell „Gastfamilien für Seniorinnen und Senioren“ ist ein Wohn- und Betreuungsangebot für die älteren Menschen, die aufgrund ihres zunehmenden Hilfebedarfs ihre Lebenssituation verändern müssen. Hierzu laufen mehrere Modellprojekte unter anderem in Nordrhein-Westfalen. ${ }^{1}$

\section{Aufgabe und Ziele}

Zielgruppe sind ältere Menschen, die nicht mehr in der eigenen Häuslichkeit verbleiben können, Pflegebedürftige sowie Personen, die eine Rund-um-die-Uhr-Betreuung benötigen oder die an einer gerontopsychiatrischen Erkrankung (z. B. Depression, Demenz) leiden.

Wesentliche Akteur(inn)e(n) dieser Wohn- und Betreuungsform sind der Träger des Projekts, zum Beispiel ein Verband der freien Wohlfahrtspflege, das von dem Projektträger gestellte Familienpflegeteam und die Gastfamilie - in Form von Familien, Einzelpersonen und Lebensgemeinschaften. Das Familienpflegeteam des Projektträgers gewinnt die Gastfamilie, bereitet sie auf die Aufnahme vor, schult sie und begleitet kontinuierlich die Arbeit. ${ }^{2}$

Das Leben in der Gastfamilie soll den älteren Menschen eine ihren Bedürfnissen entsprechende, familienbezogene individuelle Betreuung gewährleisten. Ziel des Angebotes ist es, diesem Seniorenkreis ab 65 Jahren durch die Vorteile einer persönlichen häuslichen Atmosphäre die Teilnahme an gemeinsamen alltäglichen Aktivitäten zu ermöglichen und dadurch die selbständige Lebensführung soweit wie möglich zu erhalten. Die Pflege in der Gastfamilie stellt dabei eine besondere Form des betreuten Wohnens dar. Anstelle einer sonst erforderlichen Hilfe in einer Pflegewohngruppe oder in einer stationären Pflegeeinrichtung soll der ältere Mensch Begleitung in der Gastfamilie finden. In einer Gastfamilie werden in der Regel nur eine Person, in Ausnahmefällen höchstens zwei Personen (z.B. Ehepaare) aufgenommen. ${ }^{3}$
Die Eignung sowohl der Gastfamilie als auch des älteren Menschen zur Durchführung der Familienpflege wird in der Regel im Rahmen eines kurzen Aufenthalts („Probewohnen“) geprüft.

Zwischen der Gastfamilie und dem älteren Menschen darf kein Verwandtschaftsverhältnis ersten oder zweiten Grades bestehen. Sofern eine gesetzliche Betreuung angezeigt ist, wird diese nicht von der Gastfamilie wahrgenommen. ${ }^{4}$

Als Entgelt erhalten die Gastfamilien pro Monat ca. 800 bis 1.680 Euro - abhängig von der Pflegestufe - für Unterkunft, Verpflegung und Betreuung. Das Entgelt ist von dem älteren Menschen aus seinem eigenen Einkommen oder Vermögen, ergänzt durch Leistungen der Pflegeversicherung bzw. des Sozialhilfeträgers, zu zahlen. ${ }^{5}$

Vorbilder für diese neue Wohn- und Betreuungsform sind Erfahrungen der Projektträger mit psychisch erkrankten Menschen oder seelisch behinderten Jugendlichen in der Familienpflege. $^{6}$

\section{Rechtliche Einordnung}

Bei dieser Wohnform handelt es sich um ein sogenanntes „Betreutes Wohnen“ im Sinne des $\mathbb{9} 98$ Absatz 5 SGB XII.

Zwischen der Gastfamilie und dem älteren Menschen entsteht ein Vertragsverhältnis, ebenso wie zwischen der Gastfamilie und dem das Projekt betreuenden Pflegeverband, der wiederum einen Vertrag mit dem älteren Menschen schließt. In diesen Betreuungsvereinbarungen werden die Einzelheiten zur Pflege, Versorgung, Betreuung und Begleitung festgelegt. Der jeweilige Sozialhilfeträger schließt mit dem Projektträger nach $\ 75$ SGB XII eine Vereinbarung über die Leistungen und Finanzierung des Familienpflegeteams, der Gastfamilie und die Leistungsgewährung gegenüber dem älteren Menschen. ${ }^{7}$ Sofern der ältere Mensch die Kosten nicht selbst tragen kann, kommt ganz oder teilweise eine Kostenübernahme durch den

1 Projekt Familienpflege der AWO Bielefeld „Gastfamilien für Seniorinnen und Senioren“, erhältlich im Internet unter <http://www. pflege-gt.de/index.php?page id=148> (Zugriff 22.3.2011); Arkade e.V. Ravensburg, erhältlich im Internet unter <http://www.suedkurier.de/region/bodenseekreis-oberschwaben/ravensburg/NeuerPlatz-zum-Leben-fuer-Senioren;art372490,3356714> (Zugriff: 22.3.2011); Herbstzeit gGmbH, Ortenaukreis, erhältlich im Internet unter 〈http://www.herbstzeit-bwf.de> (Zugriff: 22.3.2011); Wallonie, erhältlich im Internet unter <http://www.grenzecho.net/zeitung/ aktuell/artikel_detail.asp?a=2EF65BBB-76A6-419C-A38C-A56C7CE4DB19> (Zügriff: 22.3.2011).

2 Projekt Familienpflege der AWO Bielefeld, a.a.O.

3 Ebd.

4 Ebd.

5 Netzwerker e.V., erhältlich im Internet unter <http://www.netzwerker-verein.de/> (Zugriff 22.3.2011); 800 Euro Arkade e.V. Ravensburg, ebd.; 800 bis 1000 Euro bei Herbstzeit, ebd.

6 Herbstzeit, ebd.; Arkade e.V., ebd.; Behindertenwerk-MainKinzig,erhältlich im Internet unter <http://www.fr-online.de/rheinmain/hanau/gastfamilie-fuer-psychisch-kranke/-/1472866/3098344/-/index.html> (Zugriff 22.3.2011).

7 Projekt Familienpflege der AWO Bielefeld, ebd. 
Sozialhilfeträger in Betracht. Diese Kostenübernahme kann sich aus einem Beitrag zum Lebensunterhalt für den älteren Menschen (Grundsicherung, Unterkunft, Bekleidung, Barbetrag zur persönlichen Verfügung), einem Budget für die Betreuungsarbeit für die Familie als Familienbudget und einer Pauschale für die Anbahnung, Begleitung, Beratung und Krisenhilfe des Familienpflegeteams zusammensetzen und bei Pflegebedürftigkeit Leistungen der Hilfe zur Pflege enthalten. Vorrangig sind im Falle einer Pflegebedürftigkeit jeweils die Leistungen der Pflegekassen in Abhängigkeit von der Einstufung in die Pflegestufen.

Das Zusammenleben mit der Gastfamilie steht nicht unter dem Schutz von Artikel 6 Absatz 1 GG, da es dem Zusammenleben an der maßgeblichen Voraussetzung der von der staatlichen Rechtsordnung anerkannten Lebens- und Erziehungsgemeinschaft von Eltern und Kindern ${ }^{8}$ fehlt, zu der auch Pflegekinder gehören ${ }^{9}$, zumal schon strittig ist, ob hierzu auch im Haushalt lebende Großeltern zählen ${ }^{10}$. Für eine Ausweitung des Familienbegriffs besteht kein Bedürfnis, da es sich um eine von dem älteren Menschen und der Gastfamilie selbst gewillkürte neue Wohn- und Betreuungsform gewerblicher Art mithin nur um die Ausübung einer Haus- bzw. Begegnungsgemeinschaft ${ }^{11}$ - handelt, die über gesetzliche Regelungen wie Nachfolgeregelungen zum Heimgesetz ${ }^{12}$ hinreichend geschützt werden kann, nicht jedoch um die Wahrnehmung von Familienfunktionen im oben genannten Sinne.

\section{Risiken}

Die größte Herausforderung für den Gast und die Familie besteht bei der Einbindung in das Familienleben. In der Familie müssen sich deren Mitglieder mit der neuen Situation identifizieren. Ein neues Familienmitglied zieht ein und muss in das bisherige Familienleben integriert werden. Dieses ist nicht mit der Aufnahme eines Familienangehörigen oder eines Pflegekindes zu vergleichen. Bei einem Familienangehörigen bestehen in der Regel besondere Familienbande, die die Aufnahme und Integration leichter ermöglichen, zumal vielfach auch aus moralischen Gründen eine Verpflichtung gesehen wird. Die Beteiligen kennen sich in der Regel über einen sehr langen Zeitraum. Diese Umstände liegen bei der Aufnahme in eine Gastfamilie nicht vor.

Nicht zu unterschätzen sind die Anforderungen an die gegenseitige Anpassungsbereitschaft und -fähigkeit in Bezug auf die jeweiligen Lebensgewohnheiten der Gastfamilie bzw. des älteren Menschen, die in der Regel über Jahre gewachsen und daher als gefestigt anzusehen sind. Diese Umstände und eine zunehmende Pflegebedürftigkeit des älteren Menschen lassen die psychischen und physischen Belastungen in der Familie steigen. Die Belastungen im Rahmen der häuslichen Pflege sind schon bei pflegenden Angehörigen zunehmend relevant. So ist nach einer Studie der Siemens-Betriebskrankenkasse jeder sechste pflegende Angehörige von einer depressiven Episode bzw. von Depressionen betroffen. ${ }^{13}$ Insbesondere krankheitsbedingte Kommunikationsbarrieren, Mobilitätsdrang des älteren, unter Umständen zunehmend dement werdenden
Menschen sind wesentliche Belastungselemente für die pflegende Person und die anderen Familienmitglieder. Nicht ohne Grund stammt nach einer Untersuchung der Großteil der Täter(innen) bei Gewalt in der Pflege überwiegend aus dem nichtprofessionellen Bereich und weist einen verwandtschaftlichen Hintergrund auf. ${ }^{14}$

Die nur eingeschränkt vorhandene Anpassungsfähigkeit an die Lebensgewohnheiten der Gastfamilie stellt einen wesentlichen Unterschied zur Aufnahme eines Pflegekindes dar. Bei dem Wohn- und Betreuungsangebot für ältere Menschen obliegt die Aufsicht ausschließlich (der) dem Projektträger(in), die (der) insoweit einerseits in eine Garantenstellung geraten kann, andererseits außer einer Kündigung des Vertragsverhältnisses über keine Eingriffsbefugnisse verfügt. Einen zusätzlichen Schutz können die Nachfolgeregelungen zum Heimgesetz bieten. Sie erhöhen gleichzeitig auch die Anforderungen an die Ausgestaltung dieser Wohn- und Betreuungsform.

Die besonderen Anforderungen an die Gastfamilie bestätigen auch die Ergebnisse des Abschlussberichtes der RobertBosch-Stiftung zu dem Projekt „Leben in Gastfamilien für gerontopsychiatrisch erkrankte Menschen “ des Projektträgers Arkade e.V.: Etwa die Hälfte der Interessierten (20 Personen) gaben nach dem ersten telefonischen Kontakt an, kein Interesse mehr an der gerontopsychiatrischen Familienpflege zu haben. Als Gründe gaben sechs Personen die zu erwartende intensive Betreuung an, für vier weitere Personen war die in Aussicht gestellte Bezahlung im Vergleich zu der erwarteten intensiven Betreuung zu gering. Sechs Personen gaben ebenfalls an, die intensive Betreuung zu scheuen, interessierten sich aber für die psychiatrische Familienpflege für Erwachsene und Jugendliche. Weitere Gründe für ein Absehen von der Tätigkeit als Gastfamilie waren nicht geeignete Wohnungen und Ungeeignetheit für die Betreuung. ${ }^{15}$ Etwa ein Viertel der Interessierten konnte beruflich dem Pflege- bzw. Sozialbereich zugeordnet werden. ${ }^{16}$

$\mathrm{Zu}$ den Belastungen aus der Betreuung oder der Pflege des älteren Menschen kommen die Reaktionen aus dem Umfeld der Gastfamilien hinzu. Diese können von Unverständnis des Freundes- und Bekanntenkreises bis zur Bewunderung reichen. Andererseits können sich skeptische Einschätzungen zu

8 BVerfGE 80, 81, 90 (JuS 1990, 59).

9 BVerfGE 68, 176, 187, 189.

10 Offen gelassen in BVerfGE 39, 316, 326, verneint in BVerfGE 48, 327 , 339.

11 Zum Begriff: BVerfGE 80, $81 \mathrm{ff}$.

12 Landesrechtliche Regelungen, beispielsweise das Bremische Wohnund Betreuungsgesetz (BremWoBeG) 5.10.2010 (Brem. GBI. S. 509) oder das Wohn- und Betreuungsvertragsgesetz.

13 SBK, Pressemitteilung, erhältlich im Internet unter <http://www. sbk.org/presse/pressemitteilungen/detail/article/analyse-der-sbkzeigt-pflegende-angehoerige-sind-kraenker-als-andere-menschen. html> (Zugriff 22.3.2011).

14 Bremische Bürgerschaft, Drs. 17/1505, Gewalt in der Pflege, S. 6.

15 Robert-Bosch-Stiftung, Abschlussbericht der wissenschaftlichen Begleitung „Leben in Gastfamilien für gerontopsychiatrisch erkrankte Menschen“, erhältlich im Internet unter <http://www.bwfinfo.de/bwf_e4/literatur/2009_Geronto-Abschl-Bericht_Weissenau.pdf> (Zügriff 22.3.2011), S. 22.

16 Ebd. 
Beginn des Pflegeverhältnisses im Laufe der Zeit zu einer positiven Einschätzung wandeln. ${ }^{17}$

Der ältere Mensch kann im Gegensatz zu Kindern und Jugendlichen ein Vermögen besitzen, über das er, sofern keine rechtliche Betreuung vorliegt, uneingeschränkt verfügen kann. Das sich in einem guten persönlichen Verhältnis zwischen der Gastfamilie und dem älteren Menschen entwickelnde Vertrauen bzw. die Abhängigkeit birgt die Gefahr einer Vermögensverfügung zugunsten der Gastfamilie oder einzelner Mitglieder.

Die Unterbringung in der Gastfamilie ist eine Zwischenstation auf dem Weg ins Altenheim und erfordert im Falle eines Wechsels in eine stationäre Einrichtung ein erneutes Verlassen des gewohnten Umfeldes und im ungünstigen Fall den Abbruch zu dem durch die Gastfamilie aufgebauten sozialen Umfeld. Dies stellt insbesondere für den älteren Menschen eine zusätzliche psychische Belastung dar.

\section{Chancen}

Der demografische Wandel führt zu einer wachsenden Anzahl von älteren Menschen in Ein-Personen-Haushalten und zu überwiegend alleinstehenden älteren Frauen, da diese in der Regel eine längere Lebenserwartung haben. Ältere Frauen, die teilweise über kein eigenes familiäres Netzwerk mehr verfügen, das sie zur Versorgung und Pflege aufnehmen oder die Betreuung in der eigenen Wohnung sicherstellen kann.

Die Wohn- und Betreuungsform der Gastfamilie trägt dem Grundsatz „ambulant vor stationär“ gemäß $\mathbb{} 13$ Absatz 1 Satz 2 bis 5 SGB XII bzw. dem in der Pflegeversicherung in $\mathbb{} 3$ Satz 1 SGB XI verankerten „Vorrang häuslicher Pflege“ Rechnung und kann dem älteren Menschen ein selbstbestimmtes Leben und eine weitere Teilhabe am gesellschaftlichen Leben im Sinne des $\mathbb{}$ 71 SGB XII ermöglichen, mithin Aufgaben und Ziele, die von den Sozialhilfeträgern zu verfolgen sind. Der ältere Mensch erhält durch seine Gastfamilie das für ein menschenwürdiges Leben erforderliche soziale Umfeld.

Neben den genannten Finanzierungen kann durch den Sozialhilfeträger ergänzend eine Mittelgewährung zur Unterstützung der Teilhabe am gesellschaftlichen Leben nach $\mathbb{} 71$ SGB XII erfolgen. Gerade die Teilhabe am gesellschaftlichen Leben und das Leben in einem familiären und sozialen Umfeld können die Leistungsfähigkeit des älteren Menschen erhalten und einen wichtigen Beitrag gegen Vereinsamung leisten. Unbestritten bietet diese Wohnform für den älteren Menschen eine Steigerung der Lebensqualität.

Das Zusammenleben erfordert ein starkes soziales Engagement der Familie. Ein wesentlicher Vorteil dieser ambulanten Wohn- und Betreuungsform liegt in der professionellen Begleitung. Der ältere Mensch wird durch die Familie in der Regel semiprofessionell begleitet, ist aber im Gegensatz zu einem Verbleib in der eigenen Wohnung als Alleinstehende(r) nicht auf sich gestellt. Ergänzend steht die professionelle Betreuung durch die (den) Projektträger(in) zur Verfügung. Bei einem guten Betreuungs- oder Pflegearrangement ist diese Wohnform gut geeignet, die Fähigkeiten und Potentiale des älteren Menschen möglichst lange zu erhalten bzw. den Erhalt zu för- dern. Dieser Erhalt der Fähigkeiten, das rechtzeitige Erkennen eintretender Defizite und deren Ausgleich durch die Gastfamilie im Zusammenwirken mit dem Familienpflegeteam stellen die großen Vorteile gegenüber einem Leben als alleinstehende Person in der eigenen Wohnung dar. Bei der herkömmlichen ambulanten Pflege, also dem Leben in der eigenen Wohnung und gleichzeitiger Pflege durch einen ambulanten Pflegedienst, ist das Pflegepersonal nur für eine kurze Zeit des Tages zur Verrichtung der Pflegearbeit - zum Beispiel 30 Minuten - in der Wohnung des älteren Menschen. Für die restliche Zeit bleibt dieser sich selbst überlassen.

Im Idealfall findet mit Hilfe der Gastfamilie ein Casemanagement im Kleinen statt. Es liegt im Interesse des älteren Menschen und letztendlich auch im finanziellen Interesse der Kostenträger (Pflegeversicherung und Sozialhilfeträger), frühzeitig einen Unterstützungsbedarf zu erkennen, die Leistungsfähigkeit zu erhalten und zu fördern sowie die bedarfsgerechten und individuell erforderlichen Hilfen rechtzeitig einzusetzen. Hierfür bieten sich insbesondere niedrigschwellige und damit kostengünstigere Unterstützungsleistungen an. Vergleichbare Leistungen dieser Art sind bei einem Verbleib in der eigenen Wohnung auch mit Unterstützung durch ambulante Pflegedienste und eine Sozialarbeit der (des) Leistungsträgerin (Leistungsträgers) der Alten- und Sozialhilfe in dieser Individualität und Unmittelbarkeit nicht zu erbringen. Andererseits ist in der Gerontologie anerkannt, dass eine unzureichende Unterstützung des älteren Menschen in seinem alltäglichen Leben in der eigenen Wohnung zu einer Abwärtsspirale führen kann. Diese Abwärtsspirale nimmt sehr schnell zu und führt dadurch auch ohne das Vorliegen einer Notwendigkeit zur Zahlung von Existenzsicherungsleistung im Alter zu einer erheblichen finanziellen Belastung für die Sozialleistungsträger(innen) für erforderlich werdende Pflegeleistungen. Für die Träger(innen) der Sozialleistungen bietet die Versorgung in einer Gastfamilie somit einen finanziellen Vorteil gegenüber einer stationären Unterbringung in einem Altenheim. Ausweislich des Abschlussberichtes der Robert-Bosch-Stiftung lagen die monatlichen Kosten für ein Leben in einer Gastfamilie zwischen 900 Euro und 1.100 Euro (Basis 2002) und waren damit günstiger als ein gerontopsychiatrisches Heimangebot. ${ }^{18}$

Besonders für demenziell Erkrankte bietet die Gastfamilie ein Milieu ohne künstliche Freiheitsbeschränkungen und ein hohes Maß an persönlicher Förderung. Da die Gastfamilie die (den) neue(n) Mitbewohner(in) erst nach der Demenzerkrankung kennenlernt, fällt es ihr weniger schwer als den Angehörigen, mit problematischen Verhaltensweisen umzugehen. Das Leben in einer Gastfamilie stellt besonders für verhaltensauffällige gerontopsychiatrisch erkrankte Menschen eine Alternative zur Heimunterbringung dar. ${ }^{19}$

Bei der zukünftig zu erwartenden Diskrepanz zwischen Bedarf und Anzahl an pflegenden Personen, ausgelöst durch einen steigenden Bedarf an Pflegefachkräften einerseits und

\footnotetext{
17 Ebd., S. 26.

18 Ebd., S. 21.

19 Ebd., S. 45 f.
} 
einer sinkenden Anzahl von pflegenden Personen andererseits sowie einer hohen Fluktuation in den herkömmlichen Berufsfeldern der Altenpflege, kann diese Wohn- und Betreuungsform eine Alternative für Frauen darstellen, die ihren erlernten Beruf in der Pflege trotz den Belastungen und des schlechten Image abseits der traditionellen Beschäftigungsformen weiterhin ausüben wollen oder aufgrund von familiären Umständen müssen. Gleichzeitig ergibt sich auch hier ein arbeitsmarktpolitisches Instrument für eine Umschulung von Berufsrückkehrerinnen in Berufsfelder der Altenpflege.

Für die Gastfamilie ergibt sich zudem der arbeitsmarktpolitische Vorteil der Vereinbarkeit von Familie und Beruf, da erziehende Frauen in den Gastfamilien die Möglichkeit erhalten, zu Hause ein Einkommen zu erzielen, ohne ein prekäres Arbeitsverhältnis einzugehen. Durch die sozialversicherungspflichtige Tätigkeit leisten sie im Gegensatz zu vielen pflegenden Angehörigen einen Beitrag für ihre eigene Altersvorsorge. Private häusliche Pflegearrangements gehen nicht selten zu Lasten der Altersversorgung und Erwerbsintegration der pflegenden Person und damit meistens zu Lasten der pflegenden Frauen.

\section{Fazit}

Aus den vorgenannten Gründen ist das Modell der „Gastfamilien für Senioren und Seniorinnen “ gut geeignet, um für alle Beteiligten eine Win/Win-Situation darzustellen.

Die Evaluation der Robert-Bosch-Stiftung zeigt die Anforderungen, die bei der Auswahl der Gastfamilie zu stellen sind, sehr deutlich. ${ }^{20}$

Die ideale Gastfamilie verfügt über eine große Wohnung, möglichst ein Haus mit großem Garten; die Familienmitglieder besitzen ein sehr hohes Maß an sozialer Kompetenz und die pflegende Person kann eine Ausbildung in der Altenpflege oder Sozialarbeit vorweisen.

Insgesamt ist ein anspruchsvolles Konzept erforderlich, das folgende Aspekte beinhalten sollte:

Zur Minimierung der Risiken ist ein sehr gut strukturiertes Auswahlverfahren für die Gastfamilie erforderlich.

Die Gastfamilie darf die Aufnahme nicht ausschließlich aus finanziellen Gründen vornehmen, sondern sie muss die Aufgabe aufgrund der bereits benannten erheblichen Anforderungen auch aus einem erheblichen Anteil von Idealismus für die Versorgung des älteren Menschen übernehmen. Mit fortschreitendem Pflegebedarf stellt sich spätestens bei einer 24-StundenPflege die Frage nach einer noch adäquaten Entlohnung der Gastfamilie, die konzeptionell zur Verhinderung von Niedriglöhnen mit zu berücksichtigen ist.

Die finanziellen Vorteile für die Träger(innen) der Sozialleistungen beinhalten die Gefahr, dass diese bestrebt sein können, die Leistungserbringung verstärkt auf einen nichtprofessionellen oder semiprofessionellen Bereich wie einer Gastfamilie zu übertragen. Eine solche Intention als vordringliche Motivation für die Förderung dieser Wohn- und Betreuungsform ist aufgrund der Risiken und der konzeptionellen Anforderungen abzulehnen. Diese Wohn- und Betreuungsform kann und darf eine professionelle Pflege nicht ersetzen, sondern ist nur ein ergänzender Teil, der seinen wesentlichen, traditionellen Ursprung in einer Pflege durch die eigene Familie und hilfsweise in der kommunalen Altenhilfe bzw. in der Daseinsvorsorge findet.

$\mathrm{Zu}$ berücksichtigen sind weiter die sozialversicherungsund steuerrechtlichen Zusammenhänge und Fördermöglichkeiten, insbesondere nach $\mathbb{S} 35 \mathrm{a}$ EStG für diese Wohn- und Betreuungsform.

Das Modell „Gastfamilie“ beinhaltet gerade im semiprofessionellen Bereich erhebliche psychische und physische Belastungen für die betreuenden Personen, die ein sehr hohes $\mathrm{Maß}$ an Qualifizierung, professioneller Begleitung, Beratung und idealerweise Supervision erfordern. Die Gastfamilie und insbesondere die Hauptpflegeperson müssen ergänzend die Möglichkeit zu einer zumindest temporären Entlastung erhalten, beispielsweise durch Kurzzeitpflege oder durch ein ebenfalls neues Arrangement wie Tagesmütter in der Pflege.

Die sich wandelnden Bedingungen in der Altenpflege erfordern Mut zu neuen Wohn- und Betreuungsformen. Dabei müssen auch in anderen Lebensbereichen erprobte Betreuungsformen, wie das Leben in einer Gastfamilie oder die Tätigkeit als Tagesmutter oder -vater, einbezogen werden, um den sich verändernden Rahmenbedingungen gerecht werden zu können. Hierzu ist in dem Konzept eine Differenzierung nach den zu betreuenden Personengruppen und den sich daraus ergebenden Besonderheiten erforderlich.

Die Gastfamilie kann als eine bedarfsgerechte Versorgung von älteren Menschen mit Migrationshintergrund, die zunehmend in ein pflegebedürftiges Alter kommen, ausgestaltet werden. Durch die Versorgung in einer Gastfamilie mit vergleichbarem Migrationshintergrund kann eine kultursensible und durch das Familienpflegeteam professionell begleitete Betreuung und Pflege im Alter erfolgen, wenn herkömmliche Versorgungsformen aufgrund der kulturellen Unterschiede bei dem älteren Menschen mit Migrationshintergrund auf Ablehnung stoßen. Aufgrund der Erfahrungen mit einer Familienpflege für psychisch erkrankte Personen kann die Wohn- und Betreuungsform auch eine geeignete Versorgungsform für Menschen mit Behinderung sein - insbesondere mit geistigen Behinderungen -, die ebenfalls zunehmend das Seniorenalter erreichen. Der Vorteil liegt in dem Verbleib im gewohnten persönlichen Umfeld der Gastfamilie und einem erleichterten Wechsel in die Rente unter Aufbau einer altersgerechten Tagesstrukturierung. Zusätzliche staatliche Programme, sogenannte Seniorenmodule für Menschen mit Behinderungen, können dadurch entfallen.

Das Modell „Gastfamilie für Senioren und Seniorinnen“ muss die große Chance zur Bündelung der Vorteile der familiären Pflege mit denen der professionellen Pflege für die persönliche Versorgung des älteren Menschen nutzen, kann eine professionelle Pflege jedoch nicht vollständig ersetzen.

$20 \quad$ Ebd., S. $22 \mathrm{ff}$. 